\title{
DNA accessibility is not the primary determinant of chromatin- mediated gene regulation
}

Răzvan V. Chereji ${ }^{1,2}$, Peter R. Eriksson ${ }^{1,2}$, Josefina Ocampo ${ }^{1,2,3}$ and David J. Clark ${ }^{{ }^{*}}$

${ }^{1}$ Division of Developmental Biology, Eunice Kennedy Shriver National Institute for Child Health and Human Development, National Institutes of Health, Bethesda MD 20892, USA

${ }^{2}$ These authors contributed equally to this work.

${ }^{3}$ Current address: Instituto de Investigaciones en Ingeniería Genética y Biología Molecular "Dr. Héctor N. Torres" (INGEBI-CONICET), Buenos Aires, Argentina.

*Correspondence: Building 6A, Room 2A02, 6 Center Drive, National Institutes of Health, Bethesda MD 20892; clarkda@mail.nih.gov, Tel. (301) 496-6966.

Running title: DNA accessibility in eukaryotic cells 


\begin{abstract}
DNA accessibility is thought to be of major importance in regulating gene expression. We test this hypothesis using a restriction enzyme as a probe of chromatin structure and as a proxy for transcription factors. We measured the digestion rate and the fraction of accessible DNA at all genomic AluI sites in budding yeast and mouse liver nuclei. Hepatocyte DNA is more accessible than yeast DNA, consistent with longer linkers between nucleosomes, and indicating that nucleosome spacing is a major determinant of accessibility. DNA accessibility varies from cell to cell, such that essentially no sites are accessible or inaccessible in every cell. AluI sites in inactive mouse promoters are accessible in some cells, implying that transcription factors could bind without activating the gene. Euchromatin and heterochromatin have very similar accessibilities, suggesting that transcription factors can penetrate heterochromatin. Thus, DNA accessibility is not likely to be the primary determinant of gene regulation.
\end{abstract}




\section{INTRODUCTION}

Genomic DNA is packaged into chromatin, which is composed of regularly spaced nucleosomes. Human and mouse cells contain relatively open euchromatin (similar to yeast chromatin) and extremely compact heterochromatin (Becker et al. 2016; Allshire and Madhani 2018). Most genes located in heterochromatin are completely repressed (Becker et al. 2016). Controlling the accessibility of DNA to transcription factors is thought to be of major importance in regulating gene activation, primarily through precise positioning of a nucleosome over regulatory elements such as promoters and enhancers, blocking access to transcription factors (Fig. 1A). Activation is thought to occur when an ATP-dependent chromatin remodeler removes the blocking nucleosome, allowing transcription factors to bind, although precisely how remodelers are targeted to regulatory elements is still unclear (Voss and Hager 2014; Venkatesh and Workman 2015).

DNA accessibility may also be modulated at higher levels of chromatin structure (Fig. 1A): linker histone-dependent condensation of the chromatin filament may limit access to linker DNA between nucleosomes. Furthermore, large-scale compaction may occlude transcription factors from heterochromatin domains, perhaps involving liquid droplet phase separation (Larson et al. 2017). If DNA accessibility is the primary determinant of gene regulation, then it is crucial for repression that critical regulatory elements are blocked in all cells in a population. Otherwise, there would be inappropriate gene activation in some cells.

The accessibility model described above is appealing, but it has not yet been tested using a fully quantitative genome-wide assay. MNase-seq data are difficult to quantify because micrococcal nuclease destroys the DNA as digestion proceeds. Furthermore, nucleosomes are digested at different rates depending on the sequences they contain, resulting in apparently different relative occupancies as digestion proceeds (Chereji et al. 2017; Schwartz et al. 2018). MNase-seq data are typically normalized to the genomic average and relative nucleosome occupancies are estimated, although they are still subject to the caveat above. Three other valuable methods, DNase-seq (John et al. 2011), ATAC-seq (which uses a transposase) (Schep et al. 2015) and RED-seq/NA-Seq (which use restriction enzymes) (Gargiulo et al. 2009; Chen et al. 2014) report the relative accessibilities of open regions in chromatin. Small DNA fragments excised from accessible DNA sequences (typically regulatory elements) are isolated and sequenced. However, the rest of the genome is excluded from the analysis because it is still 
present as very long DNA molecules. Consequently, the signal and relative amounts of each accessible element depend on the extent of digestion, allowing only relative measurements. Since these methods sequence only the small fraction of accessible DNA fragments, they are only semi-quantitative.

We have adapted the restriction enzyme protection assay to measure accessibility (Linxweiler and Hörz 1984; Fascher et al. 1990; Jack and Eggert 1990; Archer et al. 1991; Verdin et al. 1993; Wallrath and Elgin 1995; Shen et al. 2001). This assay measures both the absolute accessibility of the DNA and the rate at which accessible sites are cut. It has been used in vitro to monitor nucleosome reconstitution (Zheng and Hayes 2003), to detect nucleosome shifts (Studitsky et al. 1994) and to measure the activities of chromatin remodeling enzymes (Tsukiyama and $\mathrm{Wu} 1995)$. It depends on the fact that restriction enzymes are essentially unable to cut their cognate sites within a nucleosome (Linxweiler and Hörz 1984; Polach and Widom 1995). Restriction enzymes cut nucleosomal DNA $10^{2}-10^{5}$ times slower than linker DNA, with faster rates for DNA just inside the nucleosome, as it is more likely to dissociate transiently from the histone octamer (Polach and Widom 1995; Chereji and Morozov 2014). These large rate differences result in a plateau in the digestion, corresponding to the fraction of accessible DNA (Fig. 1B; Supplemental Text).

Here, we use the restriction enzyme protection assay to measure the accessibility of a large number of specific sites throughout the genome in nuclei from budding yeast and mouse liver. We show that essentially no sites are blocked in every cell or accessible in every cell, demonstrating a high degree of cell-to-cell heterogeneity, and inconsistent with nucleosome block models. In addition, we find that DNA in budding yeast chromatin is less accessible than DNA in mouse hepatocyte chromatin, which is explained by the shorter nucleosome spacing in yeast and suggests that nucleosome spacing is the primary determinant of DNA accessibility. Mouse hepatocyte euchromatin and heterochromatin have very similar absolute DNA accessibilities and are penetrated at very similar rates, indicating that heterochromatin does not prevent restriction enzymes from accessing their sites and presumably would not exclude transcription factors either. 

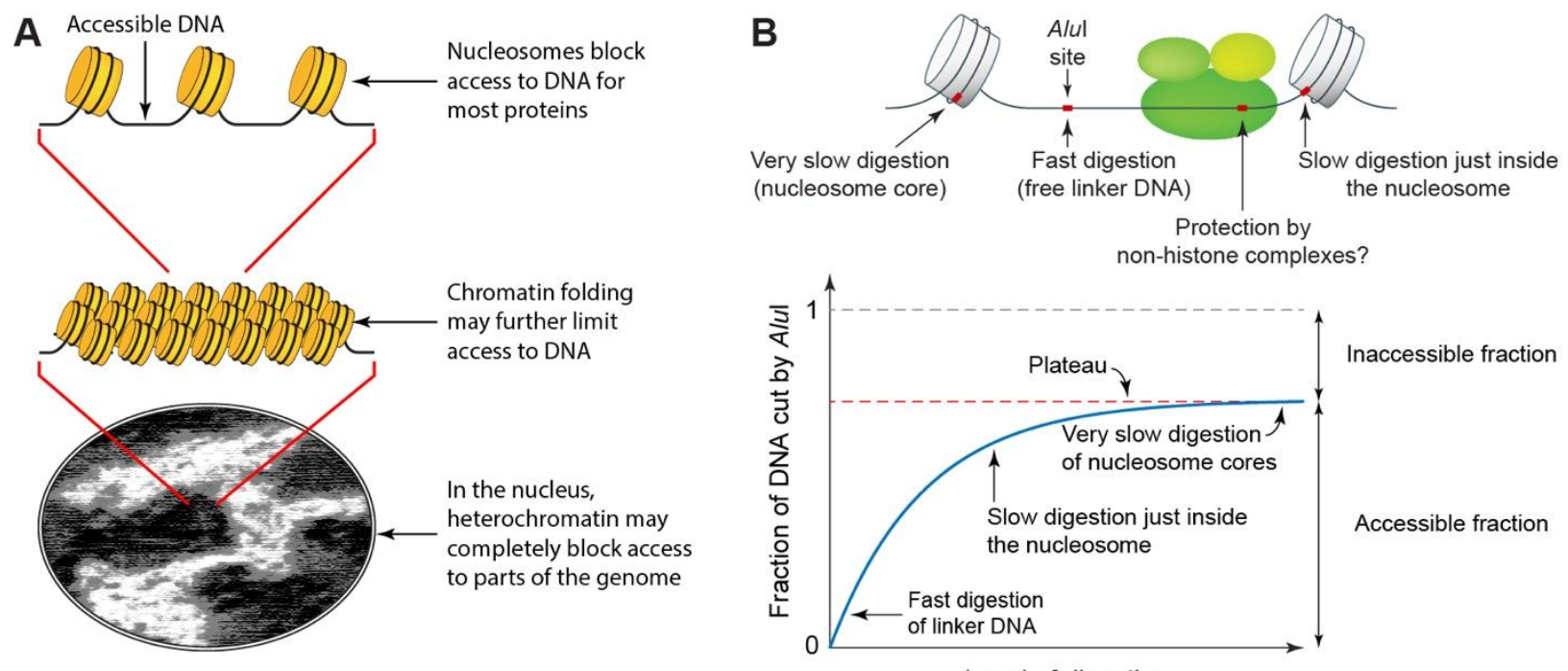

Level of digestion

Figure 1. A fully quantitative assay for DNA accessibility in chromatin. (A) DNA accessibility may depend on the degree of chromatin compaction. (B) Principle of the restriction enzyme protection assay.

\section{RESULTS}

A fully quantitative measure of DNA accessibility: qDA-seq. We used the restriction enzyme AluI to measure both the absolute DNA accessibility (i.e. the fraction of the DNA that is accessible to $A l u \mathrm{I}$ ) and the initial rate at which these accessible sites are cut. This simple method, which we term "quantitative DNA accessibility” assay (qDA-seq), involves treating nuclei with a restriction enzyme at different concentrations, sonicating the DNA into small fragments, followed by paired-end sequencing. The sonication step is necessary because the AluI digest contains many long DNA fragments derived from protected chromatin, which are not suitable for Illumina sequencing.

$A l u \mathrm{I}$ cuts the sequence $\mathrm{AG} \mid \mathrm{CT}$ to yield blunt ends. The yeast genome has $\sim 40,000 \mathrm{AluI}$ sites; the mouse genome has $\sim 12.6$ million sites. After sequencing, we calculate the fraction of DNA molecules cut at each $A l u \mathrm{I}$ site as a function of $A l u \mathrm{I}$ concentration up to $\sim 50 \mathrm{nM}$. The accessible fraction is measured by counting the number of DNA molecules with an end corresponding to a specific genomic AluI site as a fraction of all DNA molecules containing the same site. A direct comparison of accessible fractions in different cell types is possible; critically, no normalization is necessary. We note that although most of the protection observed is likely to be due to nucleosomes, the nucleosome may not be the only complex that is resistant to restriction enzymes. Such complexes would have to be stable enough to protect an AluI site during the entire incubation (20 minutes). 
DNA accessibility in yeast varies from cell to cell. To avoid potential complications due to increased accessibility of replicating DNA, we arrested haploid yeast cells in the G1 phase of the cell cycle using $\alpha$-factor. Nuclei were digested with increasing concentrations of $A l u \mathrm{I}$ and the expected plateau was observed at essentially all AluI sites (see below). It is important to note that only one copy of a unique genomic sequence is present in each cell, because the cells are haploid. Therefore, the plateau value indicates the fraction of cells in which a particular unique AluI site is accessible. Each site is accessible in some cells and inaccessible in the other cells.

We present the $A R G 1$ gene as an example (Fig. 2A). Digestion at an AluI site (site 2; Fig. 2A) just inside the -1 nucleosome reaches a plateau at $\sim 45 \%$ cut, indicating that this site is accessible in $\sim 45 \%$ of the cells and inaccessible in the remaining $\sim 55 \%$ of cells. A neighbouring $A l u$ I site (site 3) located close to the upstream border of the nucleosome-depleted region (NDR) at the $A R G 1$ promoter is more accessible, at $\sim 50 \%$. In contrast, all three $A l u \mathrm{I}$ sites in the $A R G 1$ coding region (sites 4, 5 and 6 ; located within the $+2,+3$ and +7 nucleosomes respectively) have lower accessibilities $(\sim 15-20 \%)$, suggesting that nucleosome occupancy is higher on the coding region ( $\sim 75-80 \%)$, consistent with MNase-seq data (Fig. $2 \mathrm{~A})$, and indicating that these sites are accessible in only 1 in 4 or 5 cells. The $A l u \mathrm{I}$ site in the YOLO57W promoter downstream of $A R G 1$ (site 8 ) is much more accessible, but the digestion still reaches a plateau at $\sim 60 \%$, indicating that this site is protected in $\sim 40 \%$ of cells, even though the MNase-seq data show that it is located within a deep NDR, predicting a nucleosome occupancy close to 0 (Fig. 2A). Instead, we attribute this protection to non-histone proteins stably bound at the $Y O L 057 \mathrm{~W}$ promoter in $\sim 40 \%$ of cells (Chereji et al. 2017). Importantly, a plateau is reached at all AluI sites, indicating that each site is accessible in some cells and protected in the remaining cells.

For genome-wide analysis of the data, we superimposed the plots for all $\sim 40,000 \mathrm{AluI}$ sites (Fig. 2B). A plateau is reached at a median accessibility of $\sim 22 \%$, indicating that the median AluI site is inaccessible in $\sim 78 \%$ of cells. The data range shows that $90 \%$ of $A l u \mathrm{I}$ sites are cut in only $\sim 9 \%-55 \%$ of cells (Fig. 2B). This observation indicates that yeast cells are very heterogeneous in DNA accessibility. To gain more insight, we divided the AluI sites into gene body sites and promoter sites (Fig. 2C). The median accessibility in gene bodies is very similar to that for all sites $(\sim 20 \%)$, because gene bodies account for a very large fraction of the yeast genome. If we make the simple assumption that a nucleosome protects $147 \mathrm{bp}$ of every $165 \mathrm{bp}$ (the average nucleosome spacing in yeast (Thomas and Furber 1976)), the predicted protection is 
$89 \%(147 / 165)$, which is higher than observed ( $80 \%)$, suggesting that there may be some digestion just inside the nucleosome as observed in vitro (Polach and Widom 1995), or that there may be occasional gaps in the nucleosomal arrays. In the former case, a value of $80 \%$ is consistent with a protected inner nucleosome core of $132 \mathrm{bp}(80 \%$ of $165 \mathrm{bp})$, suggesting that the outer $\sim 7$ bp on both sides of the nucleosome are vulnerable to AluI.

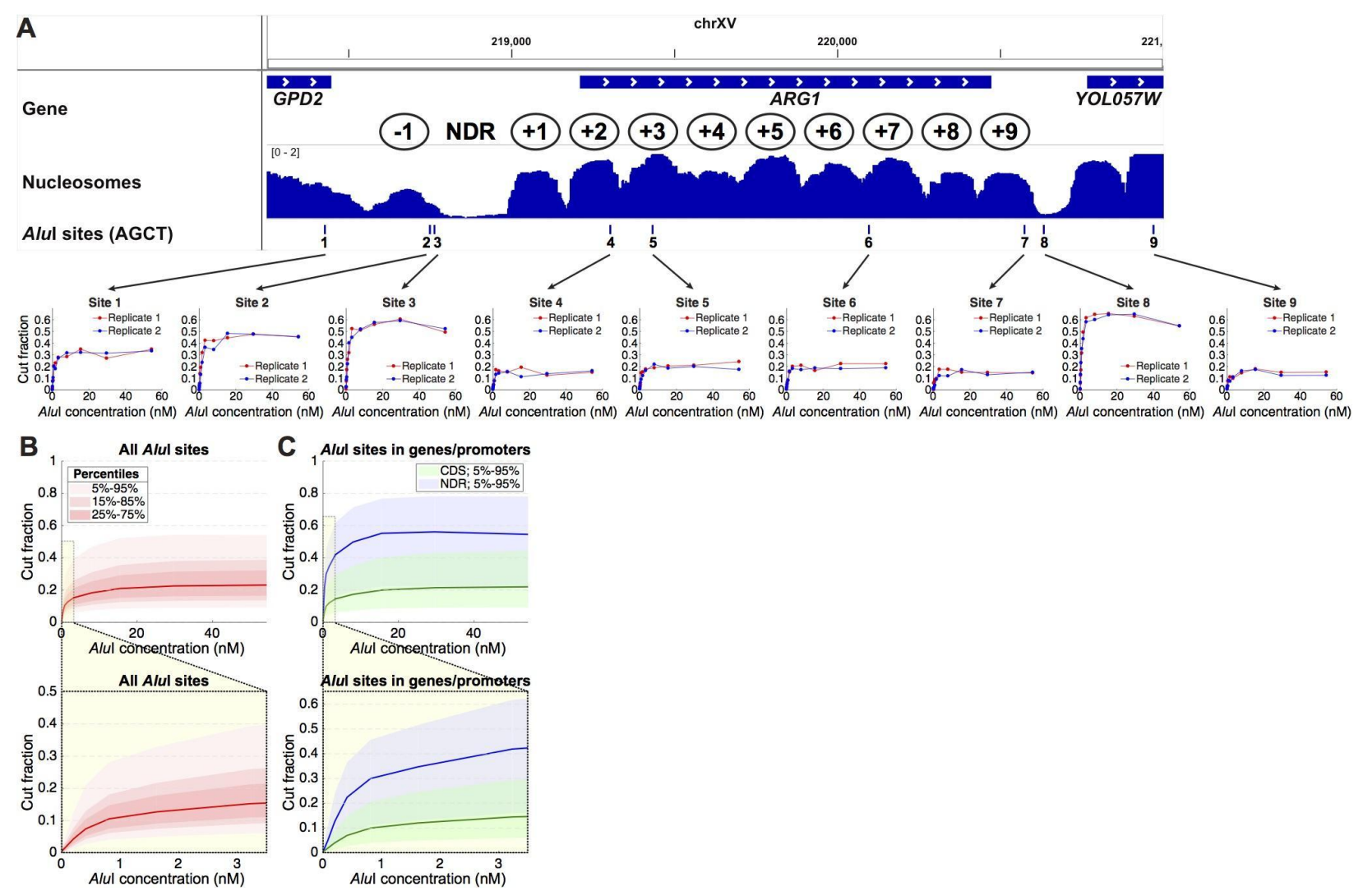

Figure 2. DNA accessibility in yeast varies from cell to cell. (A) AluI accessibility of the $A R G 1$ gene in arrested haploid yeast cells. Upper panel: Nucleosome occupancy (MNase-seq data (Ocampo et al. 2016)) in wild type cells normalized to the genomic average $(=1)$. Ovals indicate approximate nucleosome positions. Lower panel: AluI digestion at each of nine sites (data for two independent experiments are shown). The plateau value is a measure of the fraction of cells in which the AluI site is accessible. Each site is accessible in some cells and inaccessible in the rest. (B) Digestion kinetics for all $\sim 40,000 \mathrm{AluI}$ sites as a function of $[A l u \mathrm{I}]$ (11 digestion points) for haploid cells arrested with $\alpha$-factor. Red line: median level of digestion. Pink shading indicates data ranges: the lightest pink area includes $90 \%$ of the AluI sites (i.e. the 5\%-95\% data range, which excludes the 5\% of AluI sites that are the least cut and the $5 \%$ of sites that are the most cut). Lower panel: Initial stages of digestion. (C) Kinetics for AluI sites in gene bodies (between start and stop codons) and promoter NDRs defined using the positions of the +1 and -1 nucleosomes (Chereji et al. 2018). Blue line: median level of digestion in NDRs; green line: median level of digestion in gene bodies. 
The median accessibility of promoters, defined by their NDRs, is $\sim 53 \%$ (range: $90 \%$ of sites cut in $22 \%-78 \%$ of cells), which is much higher than in gene bodies, and consistent with nucleosome depletion. However, digestion within the NDR still reaches a plateau, indicating the presence of stable complexes protecting the NDR in about half of the cells, presumably corresponding to non-histone barrier complexes, as discussed above. Estimation of AluI digestion rates at accessible sites in promoter NDRs and genes, assuming first order kinetics (see Supplemental Text), indicates that NDR sites are digested only $\sim 1.3$ times faster than linker DNA sites in gene bodies (Fig. 2B, C; Supplemental Fig. S1).

\section{Imperfect nucleosome positioning can account for cell-to-cell heterogeneity in DNA}

accessibility. We plotted the mean $A l u \mathrm{I}$ accessibility for all $\sim 5,000$ yeast genes as a function of distance from the first $(+1)$ nucleosome on the gene, which typically covers the transcription start site (TSS) in yeast (Mavrich et al. 2008) (Fig. 3A). The extent of digestion as a function of AluI concentration is shown. In the absence of $A l u \mathrm{I}$, there is a background of $\sim 1 \%$ cut, corresponding to random fragmentation of the DNA at an $A l u \mathrm{I}$ site by sonication. Digestion increases with increasing $A l u \mathrm{I}$ concentration up to $\sim 15 \mathrm{nM}$, beyond which there is no more digestion. There is a strong peak at the NDR, with a maximum mean value of $\sim 55 \%$ cut. Promoters are more accessible than gene bodies, but a resistant complex is present in about half of the cells.

In gene bodies, an oscillatory pattern is observed around a mean value of $\sim 25 \%$ accessibility, anti-correlated with phased nucleosomes observed in MNase-seq data, such that the $A l u \mathrm{I}$ peaks coincide with linkers and the $A l u \mathrm{I}$ troughs coincide with nucleosomes. The amplitude of this oscillation provides a quantitative estimate of the degree of phasing. Perfectly phased nucleosomal arrays (i.e., each nucleosome occupies an identical position in every cell) predict $100 \%$ cutting at AluI sites in linkers (i.e., cut in all cells) and 0\% cutting at nucleosomal sites (i.e., blocked in all cells). In fact, the oscillations are relatively weak: the average probability of cutting an AluI site located at the +1 nucleosome position is $\sim 15 \%$, compared to $\sim 35 \%$ for AluI sites in linkers. Thus, the +1 nucleosome is shifted or absent in $\sim 15 \%$ of cells. These data can be explained by a model in which regularly spaced nucleosomes are positioned slightly differently in different cells, such that an AluI site is protected in $\sim 80 \%$ of cells and located in an accessible linker in $\sim 20 \%$ of cells (Fig. 3B). Similarly, promoters are blocked by a stable complex in about half of the cells. 

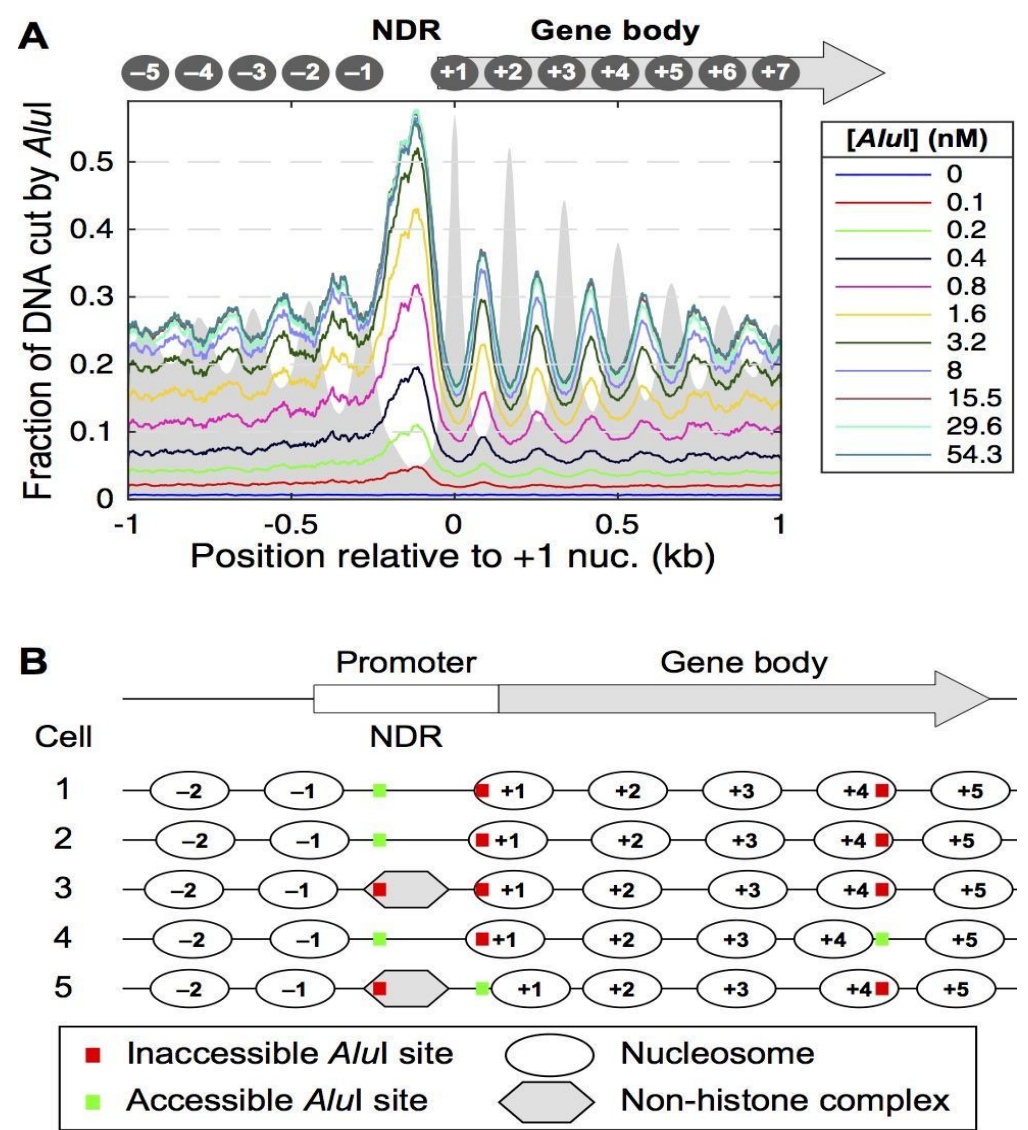

Figure 3. Genomic analysis of AluI accessibility reveals imperfect nucleosome phasing in yeast.

(A) Mean accessibility as a function of distance from the center of the +1 nucleosome (defined by Chereji et al. 2018) on all $\sim 5,000$ yeast genes. (B) Heterogeneous nucleosome positioning model to explain the $A l u \mathrm{I}$ accessibility data. On a typical gene, nucleosomes are positioned slightly differently in each cell such that a particular $A l u \mathrm{I}$ site is inside a nucleosome in one cell and in a linker in another cell. The cartoon shows the nucleosome positions on a gene in five different cells. An Alu $\mathrm{I}$ site in the coding region is in the linker (accessible) in only one cell out of five ( $20 \%$ accessibility), whereas an AluI site in the promoter NDR is accessible in three out of five cells (60\% accessibility). The observed average values are $\sim 25 \%$ in the coding region and $\sim 55 \%$ in the NDR (see A).

\section{Heavy transcription correlates with increased DNA accessibility of yeast gene bodies. We}

treated exponentially growing yeast cells with 3-aminotriazole (3AT), which induces the amino acid starvation response mediated by the Gcn 4 transcription factor (Hinnebusch and Natarajan 2002). We have shown previously that 3 AT induces heavy transcription of ARG1, HIS4 and a few other genes, resulting in chromatin disruption and loss of nucleosome occupancy over the coding region and flanking regions (Cole et al. 2014). As expected, the AluI accessibility of the ARG1 and HIS4 gene bodies increases after 3AT treatment, whereas the accessibility of AluI sites in GAL1, which is not induced by $3 \mathrm{AT}$, is unaffected (Supplemental Fig. S2). We also note 
that growing cells and $\alpha$-factor arrested cells have similar DNA accessibilities at the global level, suggesting that replication does not have a strong effect on global accessibility (Supplemental Fig. S2; compare with Fig. 3A).

The mouse hepatocyte genome is more accessible than the yeast genome. We performed the same experiment using mouse liver nuclei. AluI digestion resulted in a plateau at a median accessibility of $\sim 34 \%$ (Fig. 4A). Thus, the mouse hepatocyte genome is more accessible than the yeast genome ( $\sim 22 \%$; Fig. $2 B)$. Although this observation seems counter-intuitive, given that the yeast genome is very active and lacks heterochromatin, it is consistent with the longer average nucleosome spacing in hepatocytes ( $\sim 195$ bp; van Holde 1989$)$ relative to yeast ( $\sim 165$ bp). More insight is obtained by examining the chromatin structure in the vicinity of the average mouse promoter after alignment of all $\sim 25,000$ genes on the TSS (Fig. 4B). AluI digestion in the promoter NDR just upstream of the TSS reaches a plateau at $\sim 45 \%$ accessibility, which is higher than in the flanking regions ( $\sim 32 \%$ accessible/ $\sim 68 \%$ protected). The protection of genic DNA is consistent with a protected inner nucleosome core of $133 \mathrm{bp}(68 \%$ of $195 \mathrm{bp})$, which is essentially the same as that observed for yeast genes (132 bp). Weak nucleosome phasing is apparent downstream.

Inactive mouse gene promoters are accessible in some cells. We sorted the genes according to the DNase I hypersensitivity of their promoters in mouse hepatocytes. This analysis revealed two classes of promoter: hypersensitive and insensitive (Fig. 4C; Supplemental Fig. S3A) (Chereji and Clark 2018). After sorting using the same gene order, nucleosome positioning (MNase-Exoseq) data (Cole et al. 2016) and hepatocyte gene expression data show that genes with hypersensitive promoters are mostly active, with an NDR and phased nucleosomes, whereas genes with DNase I-insensitive promoters are inactive, lack phasing and have no NDR (Fig. 4C; Supplemental Fig. S3).

Analysis of the AluI data indicates that active genes show better phasing and higher NDR accessibility ( $\sim 58 \%$ ) than all genes (Fig. 4D; $c f$. Fig. 4B). On the other hand, DNA accessibility on both sides of the NDR is unchanged ( $32 \%$; Fig. 4D). In contrast, DNase I-insensitive genes are uniformly accessible $(\sim 32 \%)$, including promoters, with no evidence for an NDR or 
A
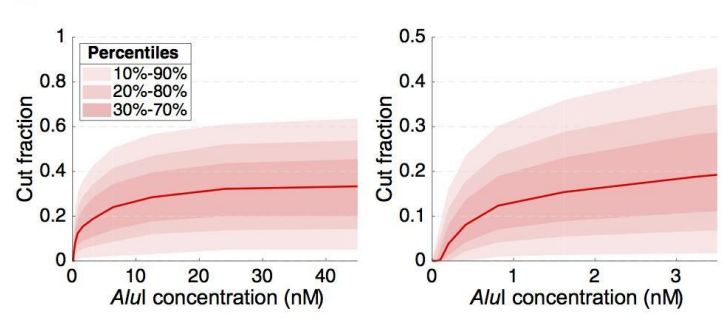

B

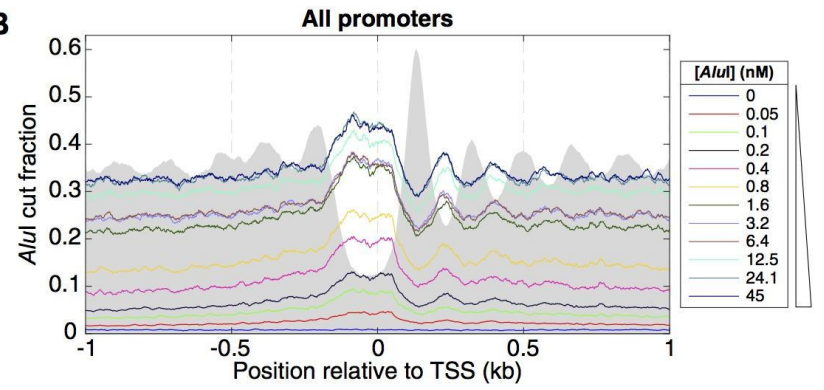

C
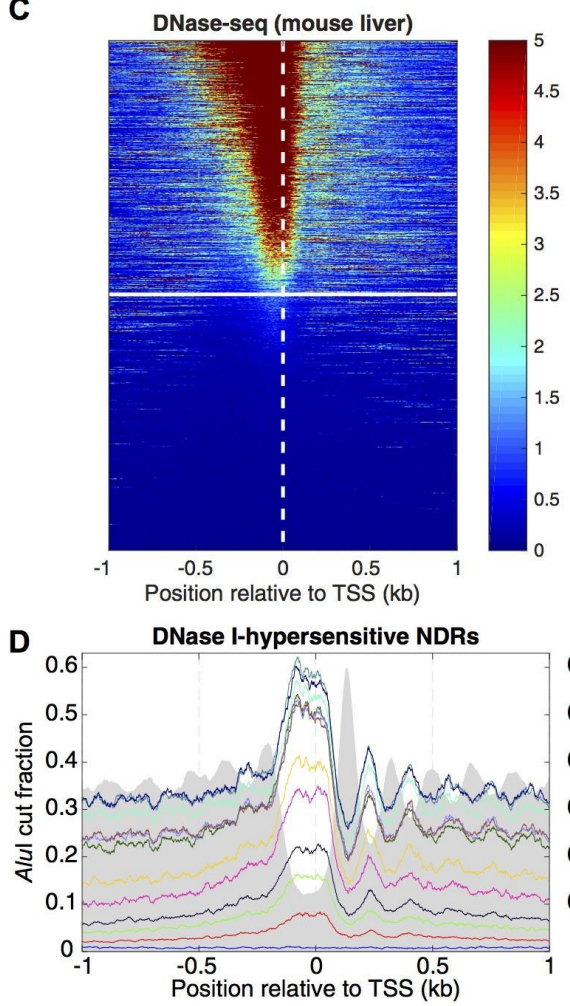
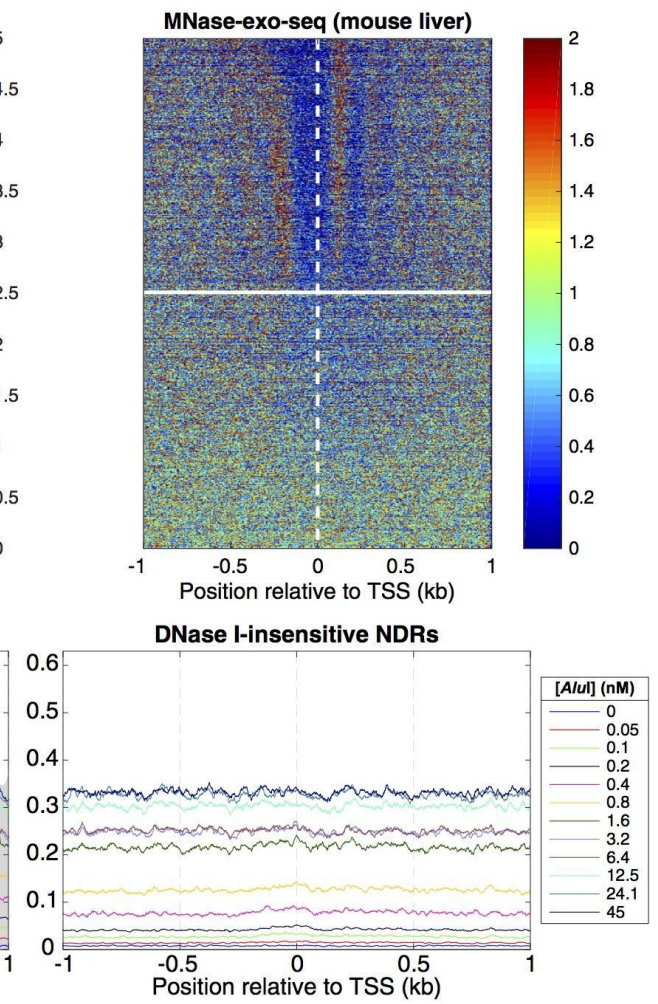

DNase l-insensitive NDRs

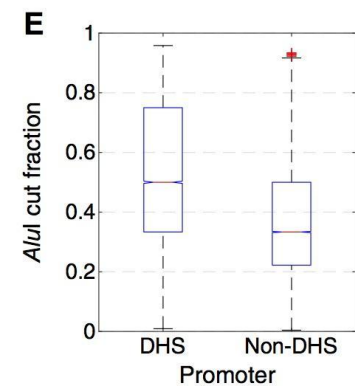

Figure 4. Inactive gene promoters are accessible to $A l u \mathrm{I}$ in some mouse liver cells. (A) AluI digestion of mouse hepatocyte nuclei (12 digestion points) Left panel: all data. Right panel: initial digestion. The data range shows that $80 \%$ of $A l u \mathrm{I}$ sites (with at least 5 reads) are cut in $5 \%-60 \%$ of cells. (B) Average $A l u \mathrm{I}$ accessibility plotted as a function of distance from the TSS on all 25,000 mouse genes. Grey area: MNase-Exo-seq data (nucleosome dyads (Cole et al. 2016)) on an arbitrary scale. (C) Heat map analysis of all 25,000 mouse genes sorted according to the DNase I hypersensitivity of their promoters in mouse hepatocytes (data from ENCODE) and aligned on the TSS: left panel: DNase I cut density; middle: nucleosome dyad positions (Cole et al. 2016); right: RNA-seq data (two biological replicates from ENCODE). The white line divides hypersensitive and insensitive promoters (defined in Supplemental Fig. S3). (D) Average AluI accessibility plotted as a function of distance from the TSS for DNase Ihypersensitive and DNase I-insensitive promoters defined in C. (E) Distribution of AluI cut fractions corresponding to sites located in promoters (region [TSS - $185 \mathrm{bp}$; TSS $+85 \mathrm{bp}$ ]), separated by DNase I hypersensitivity. The difference in DNA accessibility is highly significant (two-sided KolmogorovSmirnov test (test statistic $=0.316$ ) and two-sided t-test (test statistic $=96.288) ; \mathrm{p}$-values $<10^{-4}$; confidence interval for the difference in population means, at significance level 0.05: [0.158; 0.164]; effect size: Cohen's $d=0.755)$. 
nucleosome phasing, consistent with the nucleosome positioning data (Fig. 4C). Although inactive promoters have a lower mean accessibility $(\sim 32 \%)$ than active promoters $(\sim 58 \%)$, they are at least partly accessible in $\sim 1$ in 3 haploid genomes (i.e. on at least one allele in half of these diploid cells). The surprising accessibility of $A l u \mathrm{I}$ sites in inactive promoters probably reflects the lack of nucleosome phasing, such that regularly spaced, but unphased nucleosomal arrays result in protection of promoter $A l u \mathrm{I}$ sites in cells where they are nucleosomal and exposure in the other cells where they are in linker DNA ( $c f$. Fig. 3B).

Euchromatin and heterochromatin have very similar DNA accessibilities. We compared the accessibilities of euchromatin and heterochromatin using a 15-state epigenetic model for mouse hepatocyte chromatin derived from histone modification patterns and ChIP-seq data for Pol II and CTCF (Bogu et al. 2015) (Fig. 5). Surprisingly, the median absolute AluIaccessible fraction is similar for all 15 chromatin states (the plateau values range from $\sim 29 \%$ to $\sim 36 \%$ ), indicating that all states are accessible, including all heterochromatin states and Polycomb-repressed regions (Fig. 5A). Active promoters (states 5 and 7) and strong enhancers (states 6 and 8) were defined in the model of Bogu et al. (2015) primarily by the presence of the H3-K4me1, H3-K4me3, H3-K27ac histone marks and Pol II, whereas insulators were defined primarily by CTCF binding (state 15). All three of these regulatory elements are more accessible to AluI than the euchromatin and heterochromatin states, because they are short and dominated by an NDR, which has a higher average accessibility than the flanking chromatin (Fig. 4D, left panel). (Note that the accessibility of active promoters ( $\sim 36 \%$; Fig. $5 \mathrm{~A})$ averages lower than at promoter NDRs (58\%; Fig. 4D), because the epigenetic state model includes both the NDR and its modified flanking nucleosomes.) Most importantly, the curves for the euchromatin states (1 and 2), defined by the H3-K36me3 mark (Bogu et al. 2015), track with those for the heterochromatin states, defined by the H3-K27me3 mark (Polycomb-repressed; state 11) or by the absence of active marks (states 12 - 14), indicating that the differences between them are negligible (Fig. 5A). 

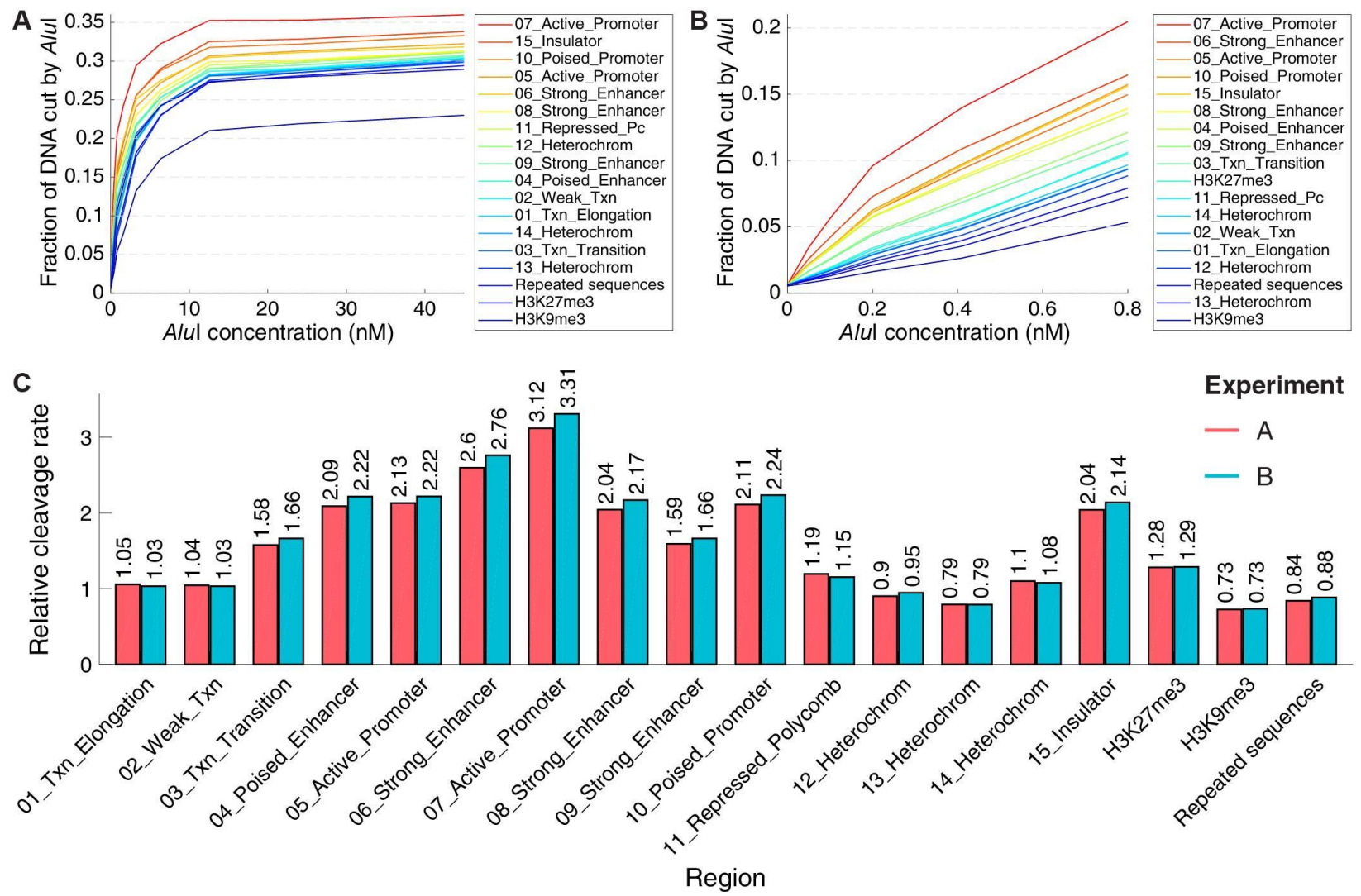

Figure 5. Heterochromatin and euchromatin have very similar DNA accessibilities in mouse hepatocytes. (A) AluI digestion kinetics for sites in the 15 epigenetic chromatin states in mouse hepatocytes defined by Bogu et al. (2015), for sites in repeated sequences (defined by Repeat Masker), and for sites marked by $\mathrm{H} 3 \mathrm{~K} 9 \mathrm{me} 3$ (constitutive heterochromatin) or $\mathrm{H} 3 \mathrm{~K} 27 \mathrm{me} 3$ (facultative heterochromatin) (data from Nocetti et al., 2019). (B) Initial rates of AluI digestion. (C) Quantitative comparison of initial digestion rates for the 15 epigenetic states defined by Bogu et al. (2015), repeated sequences and regions marked by H3K9me3 or H3K27me3. Data for biological replicate experiments A and B are shown. See Supplemental Text for details of the analysis.

The hidden Markov model used by Bogu et al. (2015) to define the various chromatin states did not include the histone marks typical of constitutive heterochromatin (H3-K9me2 and H3-K9me3). We confirmed that heterochromatin has a similar accessibility to euchromatin using two independent additional analyses. Firstly, we analyzed H3-K9me3 ChIP-seq data for adult mouse hepatocytes (Nicetto et al. 2019). These data indicate that constitutive heterochromatin defined by the $\mathrm{H} 3-\mathrm{K} 9$ me3 mark has a somewhat lower but still quite similar average absolute accessibility (plateau at $23 \%$ ) than the heterochromatin states defined by Bogu et al. (2015) ( 29\%; Fig. 5A). In the case of Polycomb-repressed/ facultative heterochromatin, analysis of ChIP-seq data for H3-K27me3 from the same source (Nicetto et al. 2019) indicates a very similar 
average absolute accessibility $(\sim 28 \%)$ to that obtained for the same mark in the Bogu model (state 11; 29\%; Fig. 5A). Secondly, we used mouse genome annotations to determine the absolute $A l u \mathrm{I}$ accessibilities of different annotated regions (Supplemental Fig. S4). We observed that all annotated regions have similar accessibilities except promoters (because of their NDRs, as discussed above). Most importantly, repeated sequences, which are strongly enriched in constitutive heterochromatin, have similar average absolute accessibility ( $\sim 29 \%)$ to that of the heterochromatin states defined by Bogu et al. ( 29\%; Fig. 5A).

Although the absolute $A l u \mathrm{I}$ accessibilities (plateau values) are similar for euchromatin and heterochromatin, it seemed possible that they might be digested at very different rates, reflecting their very different degrees of compaction. Accordingly, we analyzed the initial AluI digestion rates for accessible sites in mouse chromatin (Fig. 5B, C). Regulatory elements containing NDRs (active promoters, insulators and strong enhancers) are digested about 3 times faster than the other chromatin states ( $c f$. yeast NDRs; Supplemental Fig. S1). However, accessible sites in heterochromatin (states 11 - 14) are digested at virtually the same rate as those in euchromatin (active genes; states 1 and 2) (Fig. 5B, C). Similarly, the relative rate of AluI digestion of facultative heterochromatin as defined by Bogu et al. (2015) (state 11; 1.2-fold) is very similar to that defined by H3-K27me3 ChIP-seq data ( 1.3-fold) (Nicetto et al. 2019). The rate of digestion of constitutive heterochromatin defined by the $\mathrm{H} 3 \mathrm{~K} 9 \mathrm{me} 3$ mark $(\sim 0.7$-fold) is a little slower than for the various heterochromatin states of Bogu et al. (states 12, 13 and 14, which range from $\sim 0.8$ to $\sim 1.1$-fold), but this is a very small effect. If heterochromatin really blocks accessibility, a very large difference in AluI digestion rates for euchromatin and heterochromatin is expected, but it is not observed. Thus, DNA accessibility in mouse hepatocytes does not depend strongly on epigenetic state.

\section{DISCUSSION}

Nucleosome spacing, phasing and DNA accessibility in chromatin. Our data indicate that access to most of the genome is blocked by nucleosomes in yeast $(\sim 78 \%)$ and in mouse hepatocytes ( $\sim 68 \%$ ), consistent with average nucleosome spacings of $\sim 165 \mathrm{bp}$ and $\sim 195 \mathrm{bp}$, respectively, and a protected inner core of $\sim 133 \mathrm{bp}$. Thus, nucleosome spacing is the major determinant of absolute DNA accessibility. The inner nucleosome core completely blocks AluI but, since nucleosomes can occupy different positions in different cells (Shen et al. 2001; Cole et 
al. 2011; Small et al. 2014), all AluI sites are accessible in some cells. Thus, DNA accessibility varies from cell to cell. In yeast, most nucleosomes are phased, but nucleosome positioning is not strong enough to guarantee the inaccessibility of specific sites (Fig. 3). In mouse cells, most nucleosomes are regularly spaced, but are only well-positioned (phased) in the vicinity of active regulatory elements. Although inactive promoters have no NDR or phased nucleosomes, the nucleosomes are still regularly spaced, such that the probability of an AluI site being in the linker is determined by the average spacing.

The plateau in $A l u \mathrm{I}$ digestion indicates that protection is stable during the 20 minute digestion period, which is inconsistent with widespread nucleosome mobility in isolated nuclei, which would predict continued digestion if nucleosomes slide back and forth, alternately exposing and burying AluI sites. Nucleosomes may be more mobile in vivo due to the activities of various ATP-dependent chromatin remodelers capable of moving nucleosomes. It is likely that isolation of nuclei "freezes" the chromatin structure in the absence of ATP. Nucleosome mobility in vivo would be expected to increase the accessibility of DNA in chromatin.

DNase I hypersensitivity, ATAC-seq and $A l u I$ digestion at promoters. The hypersensitivity of active promoters to DNase I and the contrast between active and inactive promoters (compare the top and bottom halves of the heat map in Fig. 4C) suggests a very large difference in promoter accessibility between active and inactive promoters. In contrast, our AluI data indicate that the difference in absolute accessibility is quite small: $\sim 58 \%$ at the average active promoter NDR compared with $\sim 32 \%$ at inactive promoters (which have no NDR) (Fig. 4D). Similarly, our data also reveal that the difference in initial AluI digestion rates between active promoters and chromatin lacking NDRs is only $\sim 3$-fold (Fig. 5B, C). To reconcile these apparently different results, we note that DNase I hypersensitivity correlates with the presence of a promoter NDR (MNase-seq data; Fig. 4C) and that DNase I data derive from short DNA fragments released at a very early stage in digestion and so are heavily enriched for open chromatin states (NDRs); the rest of the genome is not sequenced. DNA fragments from open promoters are therefore amplified relative to the rest of the genome, resulting in a large artificial difference in accessibility between active and inactive promoters. Similar considerations apply to all methods that sequence only the initial digestion products, including typical ATAC-seq experiments. In our qDA-seq method, all of the DNA fragments are sequenced. We also perform an enzyme 
titration to prove that the accessibility limit (plateau) has been reached, but this is not possible with DNase-seq, since DNase I is not completely blocked by nucleosomes.

More generally, the DNase I hypersensitivity and Tn5 transposase (ATAC-seq) sensitivity of a promoter depend on two factors: (i) The fraction of accessible promoters: An AluI site in a specific active, open promoter is accessible in some cells but not in the others (information not provided by DNase I or ATAC-seq data). The higher this fraction is, the more DNase I or transposase cutting there would be. (ii) The width of the NDR (target size): The wider the NDR, the higher the probability of DNase I or transposase cleavage, because they are non-specific nucleases (sequence preference may be another important factor). This is clear from the heat maps in Fig. 4, in which the promoters with the most DNase I cleavage are also the ones with the widest NDRs (compare the tops of the DNase I and MNase-seq heat maps). A promoter is unlikely to contain more than one $A l u \mathrm{I}$ site and so the target size is a much less important factor.

Alternative methods for quantitative measurement of DNA accessibility involve the use of DNA methyltransferases. These enzymes can be used to methylate cytosines in accessible $\mathrm{CpG}$ or GpC dinucleotides, which can then be identified by their resistance to bisulphite conversion after sequencing (e.g. NoME-seq (Kelly et al. 2012) or MAPit (Nabilsi et al. 2014)). The methylation pattern reveals the footprints of nucleosomes and other stably bound proteins and therefore has much higher resolution than qDA-seq, although it is not as good as MNase-seq, and may be confounded by natural $\mathrm{CpG}$ methylation in the cells of higher organisms. This approach requires considerably more sequence coverage than qDA-seq and the bioinformatic analysis is much more complex. As for qDA-seq, it is necessary to demonstrate that a plateau has been reached before the inaccessible fraction can be inferred.

\section{Restriction enzymes as proxies for sequence-specific transcription factors. Since restriction} enzymes are sequence-specific, they may be considered proxies for transcription factors. Sequence-specific transcription factors must search the DNA sequence to find their cognate binding sites. The search process is facilitated by one-dimensional diffusion of the transcription factor along the DNA in non-specific binding mode, with occasional dissociation and reassociation events (Halford and Marko 2004; Woringer and Darzacq 2018). Both transcription factors and restriction enzymes find their cognate sites using this type of mechanism. When a 
transcription factor locates a cognate site, it remains bound for a relatively long time and may recruit other factors. A restriction enzyme locates a cognate site in the same way, but then cuts the DNA instead, providing a record of that binding event, which we detect and quantify in our experiment. We note that the AluI concentration range used in our experiments is in the expected range for transcription factors (up to $\sim 50 \mathrm{nM}$ ).

Mouse promoter accessibility and gene repression. The accessibility of AluI sites in inactive hepatocyte promoters implies that transcription factors can bind their cognate sites in inactive promoters in some cells, depending on whether the site is in a linker or not, and that gene inactivity is not primarily due to binding site occlusion. Although cognate sites in inactive promoters are accessible in some cells because they are located in linker DNA, an NDR is not created, suggesting that transcription factor access to DNA is insufficient for gene activation. Pioneer factors (defined as sequence-specific transcription factors capable of binding nucleosomal sites with high affinity (Zaret and Mango 2016)) may be critical, because they have the potential to bind their sites in all cells in a population, whether or not they are occupied by a nucleosome. If so, gene activation would depend on whether the pioneer factor is expressed. However, for reasons that are unclear, some pioneer factors cannot access all of their sites in vivo (Donaghey et al. 2018). An alternative model is that the key to NDR formation may be the clustering of transcription factor binding sites at promoters and enhancers; several specific transcription factors may have to be expressed and bind in concert to form an NDR before a gene can be activated. In this cooperative multi-site model (Adams and Workman 1995), single factor binding events at cognate sites in linker DNA would not be sufficient for NDR formation; all of the factors involved would need to be expressed to initiate activation.

Heterochromatin and euchromatin have similar DNA accessibilities. Our data indicate that heterochromatin is not generally less accessible than euchromatin. This conclusion is consistent with a recent quantitative analysis of MNase-seq data for human cells (Schwartz et al. 2018). We note that some accessibility is expected given that constitutive heterochromatin must be transcribed to produce the RNA required for its repression (Grewal 2010). Our data indicate that transcription factors would be expected to penetrate heterochromatin, even in its extremely compact state. However, the size of the transcription factor may be critical. Although AluI, which 
is a monomer with a relative molecular mass of $\sim 46,000$, is similar in size to many transcription factors, theoretical modeling suggests that much larger complexes may be excluded from compact heterochromatin (Maeshima et al. 2015). This is a distinct possibility given that many transcription factors are associated with large complexes.

\section{METHODS}

AluI digestion of yeast nuclei. Yeast strain YDC111 (MATa ade2-1 can1-100 leu2-3,112 trp1-1 ura3-1 (Kim et al. 2006)) was grown at $30{ }^{\circ} \mathrm{C}$ in synthetic complete (SC) medium to $\mathrm{A}_{600}=\sim 0.2$ and arrested in G1 by addition of $\alpha$-factor (FDA Core Facility) to $10 \mu \mathrm{g} / \mathrm{ml}$. Arrest was monitored by observing the appearance of the "shmoo" phenotype in a light microscope. After 2 $\mathrm{h}$, the cells were harvested by filtration and stored at $-80^{\circ} \mathrm{C}$. Spheroplasts were prepared from $\sim 100 \mathrm{~A}_{600}$ units of cells in $15 \mathrm{ml} \mathrm{SM} \mathrm{Buffer} \mathrm{(SC} \mathrm{medium} \mathrm{with} 1 \mathrm{M}$ sorbitol, $50 \mathrm{mM}$ Tris- $\mathrm{HCl} \mathrm{pH}$ 8.0, $20 \mathrm{mM}$ 2-mercaptoethanol) by digestion with 26,000 units of lyticase (Sigma L-2524) at $30{ }^{\circ} \mathrm{C}$ for $5 \mathrm{~min}$ or less. Digestion of the cell wall was monitored by measuring the $\mathrm{A}_{600}$ of $30 \mu \mathrm{l}$ cell suspension in $1 \mathrm{ml} 1 \%$ SDS and considered complete when the $\mathrm{A}_{600}$ decreased to $<10 \%$ of the initial value. For 3 AT experiments, YDC111 cells were grown to mid-log phase at $30{ }^{\circ} \mathrm{C}$ either in SC medium lacking histidine, followed by addition of 3AT (Sigma 61-82-5) to $10 \mathrm{mM}$ for $20 \mathrm{~min}$, or in SC medium (control), and stored as above. Spheroplasting of 3AT-treated cells was carried out in SM medium lacking histidine. Spheroplasts were spun down in a pre-cooled Sorvall SA600 rotor (7,500 rpm for $5 \mathrm{~min}$ at $4 \circ \mathrm{C})$ and washed once with $25 \mathrm{ml}$ cold ST Buffer (1 $\mathrm{M}$ sorbitol, $50 \mathrm{mM}$ Tris- $\mathrm{HCl} \mathrm{pH}$ 8.0). Spheroplasts were lysed by resuspension in $20 \mathrm{ml}$ cold F Buffer (18\% w/v Ficoll-PM400 (GE Healthcare 17-0300-50), 40 mM potassium phosphate, 1 $\mathrm{mM} \mathrm{MgCl}$, $\mathrm{pH}$ 6.5; protease inhibitors (Roche 05056489001) and $5 \mathrm{mM}$ 2-mercaptoethanol were added just before use). The lysate was applied to a step gradient of $15 \mathrm{ml}$ cold FG Buffer (7\% w/v Ficoll-PM400, 20\% glycerol, $40 \mathrm{mM}$ potassium phosphate, $1 \mathrm{mM} \mathrm{MgCl} 2$, $\mathrm{pH}$ 6.5, with protease inhibitors and $5 \mathrm{mM}$ 2-mercaptoethanol as above) and spun in an SA600 rotor (12,500 rpm for $20 \mathrm{~min}$ at $4 \circ \mathrm{C}$ ). The pellets (crude nuclei) were resuspended in $4.4 \mathrm{ml}$ pre-warmed $A l u \mathrm{I}$ Digestion Buffer (10 mM HEPES pH 7.5, $35 \mathrm{mM} \mathrm{NaCl}, 5 \mathrm{mM} \mathrm{MgCl}_{2}$, with protease inhibitors and $5 \mathrm{mM}$ 2-mercaptoethanol) and divided into eleven $400 \mu \mathrm{l}$ aliquots. AluI (New England Biolabs R0137 at $0.015 \mathrm{mg} / \mathrm{ml}$; MW = 46,000) was added $(0,1,2.5,5,10,20,40,100,200,400$, 
800 units), mixed thoroughly, and incubated at $25^{\circ} \mathrm{C}$ for $20 \mathrm{~min}$. Digestion was stopped by adding $50 \mu 190 \mathrm{mM}$ EDTA, 9\% SDS. Aliquots (180 $\mu \mathrm{l})$ were removed from each digest to ascertain the level of digestion; the remainders were stored at $-20{ }^{\circ} \mathrm{C}$ prior to sonication. For gel analysis, the DNA was purified by addition of $10 \mu 120 \%$ SDS, mixing, addition of $50 \mu 15 \mathrm{M}$ potassium acetate, followed by two extractions with an equal volume of chloroform, precipitation with 0.7 vol. isopropanol, and one wash with $75 \%$ ethanol. The purified DNA was dissolved in $20 \mu 10 \mathrm{mM}$ Tris- $\mathrm{HCl} \mathrm{pH}$ 8.0, 0.1 mM EDTA, $0.5 \mathrm{mg} / \mathrm{ml}$ RNase A and incubated at $37{ }^{\circ} \mathrm{C}$ for $1 \mathrm{~h}$. The DNA was analyzed in a $1 \%$ agarose gel stained with SYBR-Gold (Invitrogen S11494). For sonication, the samples were adjusted to $450 \mu \mathrm{l}$ with $180 \mu \mathrm{AluI}$ Digestion Buffer, transferred to 15-ml Sumilon TPX tubes (Diagenode C30010009) and sonicated using a Diagenode Bioruptor 300 at $4{ }^{\circ} \mathrm{C}$ and high power: 20 cycles of $30 \mathrm{~s}$ on and $30 \mathrm{~s}$ off. The DNA was purified, treated with RNase as above, purified again using Qiagen PCR purification columns (Qiagen 28106) and eluted in $50 \mu 10 \mathrm{mM}$ Tris-HCl pH 8.0, $0.1 \mathrm{mM}$ EDTA (TE(0.1)). Concentrations were determined by measuring $\mathrm{A}_{260}$. The degree of sonication was checked by analysis in a 2\% agarose gel stained with SYBR-Gold; DNA sizes ranged from $\sim 100$ to $\sim 700 \mathrm{bp}$. Prior to library preparation, the DNA was treated with repair enzymes (New England Biolabs PreCR kit M0309) and purified using Qiagen PCR purification columns as above.

AluI digestion of mouse liver nuclei. Livers were dissected from pregnant (E13.5) female $\mathrm{NIH} /$ Swiss mice and stored at $-80{ }^{\circ} \mathrm{C}$. For each experiment, a liver was thawed on ice and gently disrupted in a glass homogeniser containing $12 \mathrm{ml}$ cold Buffer A per gram liver (Buffer A: 0.34 M sucrose, $60 \mathrm{mM} \mathrm{KCl,} 15 \mathrm{mM} \mathrm{NaCl}, 15 \mathrm{mM}$ Tris- $\mathrm{HCl} \mathrm{pH}$ 8.0, $0.5 \mathrm{mM}$ spermidine-HCl, 0.15 mM spermine, $1 \mathrm{mM}$ Na-EDTA, $15 \mathrm{mM}$ 2-mercaptoethanol, and protease inhibitors as above). The homogenate was filtered through four layers of cheesecloth into a 50-ml tube on ice. Crude nuclei were collected by applying the filtrate to two 4-ml step gradients of Buffer A with $1 \mathrm{M}$ sucrose in 15-ml tubes and spinning in a Sorvall SA600 rotor at $12,500 \mathrm{rpm}$ for $15 \mathrm{mins}$ at $4{ }^{\circ} \mathrm{C}$. The supernatants were decanted and solid material on the tube sides was removed with a tissue. The nuclei were washed by gentle resuspension of both pellets in a total of $5 \mathrm{ml}$ Buffer A and spun for $5 \mathrm{~min}$ as above. The supernatant was removed, the pellet resuspended in $1 \mathrm{ml}$ Buffer A, and placed on ice. The DNA concentration was estimated by measuring the $A_{260}$ of $2 \mu 1$ nuclei in $1 \mathrm{ml} 1 \mathrm{M} \mathrm{NaOH}$. The volume of nuclei corresponding to $50 \mathrm{~A}_{260}$ units was transferred to a 1.5- 
$\mathrm{ml}$ microfuge tube and spun for $5 \mathrm{~min}$ at $14,000 \mathrm{rpm}$ at $4 \circ \mathrm{C}$. The supernatant was removed and the nuclei were resuspended in $1 \mathrm{ml}$ Mouse AluI Digestion Buffer $(0.34 \mathrm{M}$ sucrose, $60 \mathrm{mM} \mathrm{KCl}$, $15 \mathrm{mM} \mathrm{NaCl}, 15 \mathrm{mM}$ Tris- $\mathrm{HCl}$ pH 8.0, $5 \mathrm{mM} \mathrm{MgCl}_{2}, 15 \mathrm{mM}$ 2-mercaptoethanol, with protease inhibitors as above). The $\mathrm{A}_{260}$ of the diluted nuclei $(10 \mu \mathrm{l})$ was measured as above. Twelve aliquots of carefully resuspended nuclei, each containing $20 \mu \mathrm{g}$ DNA in $200 \mu \mathrm{l}$ Digestion Buffer, were titrated with $A l u \mathrm{I}$ as follows: 0, 0.3, 0.6, 1.3, 2.5, 5, 10, 20, 40, 80, 160, 320 units. The samples were mixed gently but thoroughly with a $1-\mathrm{ml}$ pipette and incubated at $37 \circ \mathrm{C}$ for $20 \mathrm{~min}$. Digestion was stopped by adding $200 \mu \mathrm{l}$ \% SDS, 20 mM EDTA, 10 mM Tris-HCl pH 8.0, mixing thoroughly and incubating for $40 \mathrm{~min}$ at room temperature to ensure complete protein removal from DNA. The extent of digestion was determined by analysis of DNA purified from $50 \mu \mathrm{l}$ of each digest in an agarose gel, after RNase treatment as above. The remaining $350 \mu \mathrm{l}$ was stored at $-20{ }^{\circ} \mathrm{C}$ prior to sonication. The samples were warmed to room temperature to dissolve precipitated SDS, the volumes were adjusted to $450 \mu \mathrm{l}$ with $100 \mu 110 \mathrm{mM} \mathrm{TE}(0.1)$ and sonicated as above. The DNA was purified as above and dissolved in $45 \mu 150 \mathrm{mM}$ Tris-HCl pH 8.0, $5 \mathrm{mM}$ EDTA, $0.1 \mathrm{mg} / \mathrm{ml}$ RNase A and incubated for $1 \mathrm{~h}$ at $37{ }^{\circ} \mathrm{C}$. The salt concentration was adjusted by addition of $5 \mu \mathrm{l} 10 \mathrm{x}$ NEB Buffer 4 and the DNA was purified using Qiagen PCR columns. DNA was eluted in $50 \mu \mathrm{TE}(0.1)$. Concentrations were measured by $\mathrm{A}_{260}$. The degree of sonication was checked by analysis in a $2 \%$ agarose gel; DNA sizes ranged from $\sim 100$ to $\sim 700$ bp.

Illumina paired-end library preparation. The Illumina paired-end adaptor was ligated to 500 ng purified sonicated $A l u$ I-digested DNA using the NEBNext Ultra DNA library kit for Illumina (New England Biolabs E7370) according to the manufacturer's instructions. The ligated DNA samples were purified without size selection using AMPure XP beads (Beckman A63880). The DNA (50-100 ng) was amplified using the Phusion Hi-Fi PCR master mix with HF buffer (New England Biolabs M0531) or the Q5 Hot Start HiFi PCR master mix (New England Biolabs E6625AA) (7 - 10 cycles). Library quality was checked in an agarose gel. Sequencing was performed using either an Illumina HiSeq-2500 or an Illumina NextSeq-500.

Bioinformatics and data analysis. Paired-end reads were aligned to the $S$. cerevisiae reference genome sacCer3, or to the M. musculus reference genome mm10, using Bowtie2 (Langmead and Salzberg 2012) with the parameters -X 5000 --very-sensitive, to map sequences up to $5 \mathrm{~kb}$ with maximum accuracy. For every AluI motif (AGCT) found in the genome, we estimated the 
fraction of nuclei in which the given motif was cleaved by $A l u \mathrm{I}, f_{c u t}=N_{c u t} /\left(N_{c u t}+N_{u n c u t}\right)$, by counting the number of reads that were cut at this site (with an end at this site), $N_{c u t}$, and the number of reads that were not cut at this site (overlapping the motif), $N_{\text {uncut }}$, using the Bioinformatics toolbox from Matlab. Because we sequenced 50 nucleotides from both ends of the DNA fragments, we discarded the $A l u \mathrm{I}$ sites which were less than $50 \mathrm{bp}$ apart, as the reads originating from cleavages at both sites were underrepresented in the properly aligned reads. GEO database data used: MNase-Exo-seq (GSE65889), DNase-seq (GSM1014183 in GSE37074) and RNA-seq (GSM2071423 and GSM2071424 in GSE78391).

\section{DATA ACCESS}

The sequencing data from this study have been submitted to the NCBI Gene Expression Omnibus (http://www.ncbi.nlm.nih.gov/geo/) under accession number GSE115693. Reviewer link:

\section{https://www.ncbi.nlm.nih.gov/geo/query/acc.cgi?token=crszoicibhuxvkd\&acc=GSE115693}

Code: https://github.com/rchereji/AluI_accessibility analysis (code from this repository will be made public before publication).

\section{ACKNOWLEDGMENTS}

We thank Gary Felsenfeld, Gordon Hager, Alan Hinnebusch, Tom Johnson, and Diego Presman for comments on the manuscript, Valya Russanova for technical support, and Phil Lee, Will Huffman and Doug Fields for mouse livers. We thank the NHLBI Sequencing Core Facility (Yan Luo, Poching Liu and Yuesheng Li) for paired-end sequencing. This study utilized the computational resources of the NIH HPC Biowulf cluster. This work was supported by the Intramural Research Program of the National Institutes of Health (NICHD).

\section{DISCLOSURE DECLARATION}

None. 


\section{REFERENCES}

Adams CC, Workman JL. 1995. Binding of disparate transcriptional activators to nucleosomal DNA is inherently cooperative. Mol Cell Biol 15: 1405-1421.

Allshire RC, Madhani HD. 2018. Ten principles of heterochromatin formation and function. Nat Rev Mol Cell Biol 19: 229-244.

Archer TK, Cordingley MG, Wolford RG, Hager GL. 1991. Transcription factor access is mediated by accurately positioned nucleosomes on the mouse mammary tumor virus promoter. Mol Cell Biol 11: $688-698$.

Becker JS, Nicetto D, Zaret KS. 2016. H3K9me3-Dependent Heterochromatin: Barrier to Cell Fate Changes. Trends Genet 32: 29-41.

Bogu GK, Vizán P, Stanton LW, Beato M, Di Croce L, Marti-Renom MA. 2015. Chromatin and RNA maps reveal regulatory long noncoding rnas in mouse. Mol Cell Biol 36: 809-819.

Chen PB, Zhu LJ, Hainer SJ, McCannell KN, Fazzio TG. 2014. Unbiased chromatin accessibility profiling by RED-seq uncovers unique features of nucleosome variants in vivo. BMC Genomics 15: 1104.

Chereji RV, Clark DJ. 2018. Major determinants of nucleosome positioning. Biophys J 114: 2279-2289.

Chereji RV, Morozov AV. 2014. Ubiquitous nucleosome crowding in the yeast genome. Proc Natl Acad Sci USA 111: 5236-5241.

Chereji RV, Ocampo J, Clark DJ. 2017. MNase-Sensitive Complexes in Yeast: Nucleosomes and Nonhistone Barriers. Mol Cell 65: 565-577.e3.

Chereji RV, Ramachandran S, Bryson TD, Henikoff S. 2018. Precise genome-wide mapping of single nucleosomes and linkers in vivo. Genome Biol 19: 19.

Cole HA, Cui F, Ocampo J, Burke TL, Nikitina T, Nagarajavel V, Kotomura N, Zhurkin VB, Clark DJ. 2016. Novel nucleosomal particles containing core histones and linker DNA but no histone H1. Nucleic Acids Res 44: 573-581.

Cole HA, Howard BH, Clark DJ. 2011. Activation-induced disruption of nucleosome position clusters on the coding regions of Gcn4-dependent genes extends into neighbouring genes. Nucleic Acids Res 39: 9521-9535.

Cole HA, Ocampo J, Iben JR, Chereji RV, Clark DJ. 2014. Heavy transcription of yeast genes correlates with differential loss of histone $\mathrm{H} 2 \mathrm{~B}$ relative to $\mathrm{H} 4$ and queued RNA polymerases. Nucleic Acids Res 42: 12512-12522.

Donaghey J, Thakurela S, Charlton J, Chen JS, Smith ZD, Gu H, Pop R, Clement K, Stamenova EK, Karnik R, et al. 2018. Genetic determinants and epigenetic effects of pioneer-factor occupancy. Nat Genet 50: 250-258.

Fascher KD, Schmitz J, Hörz W. 1990. Role of trans-activating proteins in the generation of active chromatin at the PHO5 promoter in S. cerevisiae. EMBO J 9: 2523-2528.

Gargiulo G, Levy S, Bucci G, Romanenghi M, Fornasari L, Beeson KY, Goldberg SM, Cesaroni M, 
Ballarini M, Santoro F, et al. 2009. NA-Seq: a discovery tool for the analysis of chromatin structure and dynamics during differentiation. Dev Cell 16: 466-481.

Grewal SI. 2010. RNAi-dependent formation of heterochromatin and its diverse functions. Curr Opin Genet Dev 20: 134-141.

Halford SE, Marko JF. 2004. How do site-specific DNA-binding proteins find their targets? Nucleic Acids Res 32: 3040-3052.

Hinnebusch AG, Natarajan K. 2002. Gcn4p, a master regulator of gene expression, is controlled at multiple levels by diverse signals of starvation and stress. Eukaryotic Cell 1: 22-32.

van Holde KE. 1989. Chromatin (New York, NY: Springer New York).

Jack RS, Eggert H. 1990. Restriction enzymes have limited access to DNA sequences in Drosophila chromosomes. EMBO J 9: 2603-2609.

John S, Sabo PJ, Thurman RE, Sung M-H, Biddie SC, Johnson TA, Hager GL, Stamatoyannopoulos JA. 2011. Chromatin accessibility pre-determines glucocorticoid receptor binding patterns. Nat Genet 43: $264-268$.

Kelly TK, Liu Y, Lay FD, Liang G, Berman BP, Jones PA. 2012. Genome-wide mapping of nucleosome positioning and DNA methylation within individual DNA molecules. Genome Res 22: 2497-2506.

Kim Y, McLaughlin N, Lindstrom K, Tsukiyama T, Clark DJ. 2006. Activation of Saccharomyces cerevisiae HIS3 results in Gen4p-dependent, SWI/SNF-dependent mobilization of nucleosomes over the entire gene. Mol Cell Biol 26: 8607-8622.

Langmead B, Salzberg SL. 2012. Fast gapped-read alignment with Bowtie 2. Nat Methods 9: 357-359.

Larson AG, Elnatan D, Keenen MM, Trnka MJ, Johnston JB, Burlingame AL, Agard DA, Redding S, Narlikar GJ. 2017. Liquid droplet formation by HP1 $\alpha$ suggests a role for phase separation in heterochromatin. Nature 547: 236-240.

Linxweiler W, Hörz W. 1984. Reconstitution of mononucleosomes: characterization of distinct particles that differ in the position of the histone core. Nucleic Acids Res 12: 9395-9413.

Maeshima K, Kaizu K, Tamura S, Nozaki T, Kokubo T, Takahashi K. 2015. The physical size of transcription factors is key to transcriptional regulation in chromatin domains. J Phys Condens Matter 27: 064116.

Mavrich TN, Ioshikhes IP, Venters BJ, Jiang C, Tomsho LP, Qi J, Schuster SC, Albert I, Pugh BF. 2008. A barrier nucleosome model for statistical positioning of nucleosomes throughout the yeast genome. Genome Res 18: 1073-1083.

Nabilsi NH, Deleyrolle LP, Darst RP, Riva A, Reynolds BA, Kladde MP. 2014. Multiplex mapping of chromatin accessibility and DNA methylation within targeted single molecules identifies epigenetic heterogeneity in neural stem cells and glioblastoma. Genome Res 24: 329-339.

Nicetto D, Donahue G, Jain T, Peng T, Sidoli S, Sheng L, Montavon T, Becker JS, Grindheim JM, Blahnik K, et al. 2019. H3K9me3-heterochromatin loss at protein-coding genes enables developmental lineage specification. Science 363: 294-297.

Ocampo J, Chereji RV, Eriksson PR, Clark DJ. 2016. The ISW1 and CHD1 ATP-dependent chromatin 
remodelers compete to set nucleosome spacing in vivo. Nucleic Acids Res 44: 4625-4635.

Polach KJ, Widom J. 1995. Mechanism of protein access to specific DNA sequences in chromatin: a dynamic equilibrium model for gene regulation. J Mol Biol 254: 130-149.

Schep AN, Buenrostro JD, Denny SK, Schwartz K, Sherlock G, Greenleaf WJ. 2015. Structured nucleosome fingerprints enable high-resolution mapping of chromatin architecture within regulatory regions. Genome Res 25: 1757-1770.

Schwartz U, Németh A, Diermeier S, Exler JH, Hansch S, Maldonado R, Heizinger L, Merkl R, Längst G. 2018. Characterizing the nuclease accessibility of DNA in human cells to map higher order structures of chromatin. Nucleic Acids Res.

Shen CH, Leblanc BP, Alfieri JA, Clark DJ. 2001. Remodeling of yeast CUP1 chromatin involves activator-dependent repositioning of nucleosomes over the entire gene and flanking sequences. Mol Cell Biol 21: 534-547.

Small EC, Xi L, Wang J-P, Widom J, Licht JD. 2014. Single-cell nucleosome mapping reveals the molecular basis of gene expression heterogeneity. Proc Natl Acad Sci USA 111: E2462-71.

Studitsky VM, Clark DJ, Felsenfeld G. 1994. A histone octamer can step around a transcribing polymerase without leaving the template. Cell 76: 371-382.

Thomas JO, Furber V. 1976. Yeast chromatin structure. FEBS Lett 66: 274-280.

Tsukiyama T, Wu C. 1995. Purification and properties of an ATP-dependent nucleosome remodeling factor. Cell 83: 1011-1020.

Venkatesh S, Workman JL. 2015. Histone exchange, chromatin structure and the regulation of transcription. Nat Rev Mol Cell Biol 16: 178-189.

Verdin E, Paras P, Van Lint C. 1993. Chromatin disruption in the promoter of human immunodeficiency virus type 1 during transcriptional activation. EMBO J 12: 3249-3259.

Voss TC, Hager GL. 2014. Dynamic regulation of transcriptional states by chromatin and transcription factors. Nat Rev Genet 15: 69-81.

Wallrath LL, Elgin SC. 1995. Position effect variegation in Drosophila is associated with an altered chromatin structure. Genes Dev 9: 1263-1277.

Woringer M, Darzacq X. 2018. Protein motion in the nucleus: from anomalous diffusion to weak interactions. Biochem Soc Trans 46: 945-956.

Zaret KS, Mango SE. 2016. Pioneer transcription factors, chromatin dynamics, and cell fate control. Curr Opin Genet Dev 37: 76-81.

Zheng C, Hayes JJ. 2003. Intra- and inter-nucleosomal protein-DNA interactions of the core histone tail domains in a model system. $J$ Biol Chem 278: 24217-24224. 